TRANSACTIONS OF THE

AMERICAN MATHEMATICAL SOCIETY

Volume 350, Number 1, January 1998, Pages 43-53

S 0002-9947(98)01556-6

\title{
DECOMPOSITION THEOREMS AND APPROXIMATION BY A "FLOATING" SYSTEM OF EXPONENTIALS
}

\author{
E. S. BELINSKII
}

\begin{abstract}
The main problem considered in this paper is the approximation of a trigonometric polynomial by a trigonometric polynomial with a prescribed number of harmonics. The method proposed here gives an opportunity to consider approximation in different spaces, among them the space of continuous functions, the space of functions with uniformly convergent Fourier series, and the space of continuous analytic functions. Applications are given to approximation of the Sobolev classes by trigonometric polynomials with prescribed number of harmonics, and to the widths of the Sobolev classes.

This work supplements investigations by Maiorov, Makovoz and the author where similar results were given in the integral metric.
\end{abstract}

\section{INTRODUCTION}

Let $X$ be a Banach space of functions defined on the cube $\mathbb{T}^{n}=(-\pi, \pi]^{n}$ and $2 \pi$-periodic in each variable. For $\mathbf{k}=\left(k_{1}, \ldots, k_{n}\right) \in \mathbb{Z}^{n}$, let $\mathbf{x}=\left(x_{1}, \ldots, x_{n}\right) \in$ $\mathbb{R}^{n}, \quad(\mathbf{x}, \mathbf{k})=k_{1} x_{1}+\ldots+k_{n} x_{n}$, and let $T\left(\theta_{m} ; \mathbf{x}\right)=\sum_{\mathbf{k} \in \theta_{m}} c_{\mathbf{k}} e^{i(\mathbf{k}, \mathbf{x})}$ be a trigonometric polynomial with the spectrum from the set $\theta_{m}$ of finite cardinality $m$.

Let

$$
e_{m}(f ; X) \stackrel{\text { def }}{=} \inf _{\theta_{m}} \inf _{T\left(\theta_{m}\right)}\left\|f-T\left(\theta_{m}\right)\right\|_{X}
$$

be the degree of best approximation of $f$ by polynomials with $m$ harmonics. The quantity $e_{m}\left(f ; L_{2}\right)$ originally appeared in a paper by S. B. Stechkin [St], who used it in the criterion for absolute convergence of orthogonal series. This characteristic has become popular after Ismagilov's excellent paper [Is], in which he found nontrivial estimates for $e_{m}\left(|x| ; L_{\infty}\right)$ and gave interesting and important applications to the widths of Sobolev classes. The Ismagilov method was developed in a series of papers by V. Maiorov (see, for example, [Mr1], [Mr2], [Mr3]). The probabilistic method for constructing approximating polynomials was proposed by Y. Makovoz [Mk] and the author $[\mathrm{Be} 1]$.

This paper is organized as follows. First we obtain an estimate in the space $C(\mathbb{T})$ of continuous functions of one variable and describe the method in all details. In the subsequent sections the method is used in other situations: the space $U$ of uniformly convergent Fourier series, the space $C_{A}$ of continuous analytic functions,

Received by the editors March 13, 1995.

1991 Mathematics Subject Classification. Primary 42A61.

Key words and phrases. Approximation, width.

This research was supported by the Israeli Ministry of Science and the Arts through the Ma'agara program for absorption of immigrant mathematicians at the Technion, Israel Institute of Technology. 
and the space $C\left(\mathbb{T}^{n}\right)$ of continuous functions of $n$ real variables. The proofs in these sections are less detailed, and often only necessary differences are pointed out.

Applications are given in the concluding section. As usual $C_{s}$ denotes an absolute constant.

\section{Approximation IN THE SPACE $C(\mathbb{T})$}

Let $T_{N}$ be a trigonometric polynomial of degree at most $N$, namely,

$$
T_{N}(x)=\sum_{k=-N}^{N} c_{k} e^{i k x} .
$$

Theorem 1. For every $2 \leq p<\infty$ and $1 \leq M \leq 2 N$ there exists a trigonometric polynomial $T\left(\theta_{M} ; x\right)$ with number of harmonics at most $M$, such that

$$
\left\|T_{N}(x)-T\left(\theta_{M} ; x\right)\right\|_{\infty} \leq C_{1}\left(\frac{N}{M} \log \left(1+\frac{N}{M}\right)\right)^{1 / p}\left\|T_{N}\right\|_{p},
$$

and the spectrum $\theta_{M}$ is in the segment $[-2 N, 2 N]$.

The proof is based on the following lemmas.

Lemma 1. Let $V_{2 N}(f ; x)$ be the de la Vallée-Poussin means of the function $f$. Then

$$
\left\|V_{2 N}(f ; x)\right\|_{\infty} \leq C_{2}\|f\|_{\infty} .
$$

This lemma is well known. Its proof may be found, for example, in [Z]. We note only that $V_{2 N}(f)$ is a trigonometric polynomial of degree $\leq 2 N$ and for every trigonometric polynomial $T_{N}$ of degree $\leq N$

$$
V_{2 N}\left(T_{N} ; x\right) \equiv T_{N}(x) .
$$

We denote by $\|\cdot\|_{\psi}$ the norm of the Orlicz space generated by the function $\psi(u)=e^{u^{2}}-1$ (see, for example, [Z]).

Lemma 2. For every trigonometric polynomial $T_{N}(x)$ and for every $M \leq 2 N$ there exists a trigonometric polynomial $T\left(\theta_{M} ; x\right)$, with the number of harmonics at most $M$, such that

$$
\left\|T_{N}(x)-T\left(\theta_{M} ; x\right)\right\|_{\psi} \leq C_{3}\left(\frac{N}{M}\right)^{1 / 2}\left\|T_{N}\right\|_{2},
$$

where the spectrum $\theta_{M} \subset[-N, N]$.

In the space $L_{q}(2<q<\infty)$ such an inequality was proved in [Be2], [Mk]. The proof proposed in [Bo] is based on Khinchin's inequality and can be used in this situation with minimal changes. For the sake of completeness we shall give the proof later.

Lemma 3. For each $\lambda>0$, every polynomial $T_{N} \in L_{\psi}$ can be represented as a sum of two polynomials $T_{N}=T_{2 N}^{1}+T_{2 N}^{2}$, each of degree $\leq 2 N$, such that

$$
\left\|T_{2 N}^{1}\right\|_{\infty} \leq C_{4} \lambda\left\|T_{N}\right\|_{\psi}, \quad\left\|T_{2 N}^{2}\right\|_{\infty} \leq C_{5} e^{-\lambda^{2}}\left\|T_{N}\right\|_{\psi} .
$$

For the proof, it is sufficient to truncate the polynomial $T_{N}$ at level $\lambda$ and apply the de la Vallée-Poussin operator to both sides of the equality (see Lemma 1). 


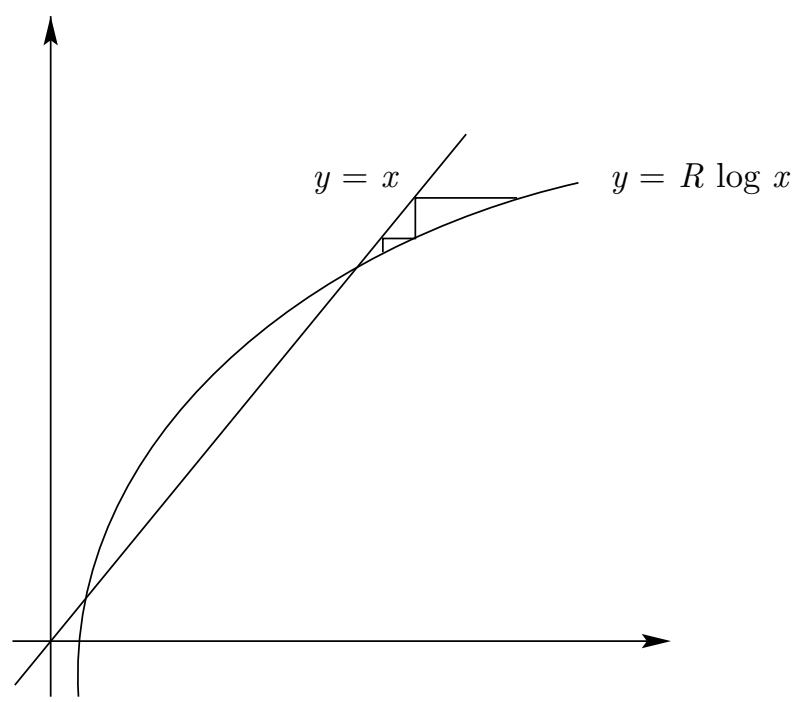

FiguRE 1

Lemma 4. For every trigonometric polynomial $T_{N}$ and for every $M \leq 2 N$ there exists a trigonometric polynomial $T\left(\theta_{M} ; x\right)$, with the cardinality of the spectrum $\leq M$, such that

$$
\left\|T_{N}(x)-T\left(\theta_{M} ; x\right)\right\|_{\infty} \leq C_{6}\left(\frac{N}{M} \log N\right)^{1 / 2}\left\|T_{N}\right\|_{2} .
$$

This can be derived from the original proof given in [Be1], as well as from the corresponding statement for the space $L_{q}(2<q<\infty)$ (see [Be2], [Mk], [Bo]), if the order of growth $C_{q} \sim \sqrt{q}$ in Khinchin's inequality is taken into account.

Proof of Theorem 1. First let $p=2$. Denote $N / M=R$ and consider the iterated sequence

$$
\begin{gathered}
a_{1}(N, M)=R \log N, \quad a_{2}(N, M)=R \log a_{1}(N, M), \ldots, \\
a_{k+1}(N, M)=R \log a_{k}(N, M), \ldots
\end{gathered}
$$

The sequence $\left\{a_{k}(N, M)\right\}$ converges to $x_{0}$, the largest root of the equation $x=$ $R \log x$. This can be easily seen from Figure 1 .

The $x_{0}$ can be estimated by $R \log R<x_{0}<2 R \log R$. We will prove that there is a number $\alpha$ such that for every $k$ and $1 \leq M \leq \alpha N$ there exists a trigonometric polynomial $T\left(\theta_{M}^{k}\right)$ such that

$$
\left\|T_{N}-T\left(\theta_{M}^{k}\right)\right\|_{\infty} \leq C_{6} \sqrt{a_{k}(N, M)}\left\|T_{N}\right\|_{2}
$$

Then after a finite number of steps we will obtain the desired estimate.

For $k=1$ the estimate (1.6) is given in Lemma 4. Assume that (1.6) holds for $k$ for all $N$; we now prove it for $k+1$. According to Lemma 2, there exists a polynomial $T\left(\theta_{M} ; x\right)$ such that

$$
\left\|T_{N}(x)-T\left(\theta_{M} ; x\right)\right\|_{\psi} \leq C_{3}\left(\frac{N}{M}\right)^{1 / 2}\left\|T_{N}\right\|_{2} .
$$


Applying Lemma 3, we find the decomposition

$$
T_{N}(x)-T\left(\theta_{M} ; x\right)=T_{2 N}^{1}(x)+T_{2 N}^{2}(x),
$$

for a parameter $\lambda$ yet to be prescribed. Using our assumption for $k$, we approximate the polynomial $T_{2 N}^{2}$ by a polynomial $T\left(\theta_{M}^{k} ; x\right)$ in the norm $L_{\infty}$. Then

$$
\begin{gathered}
\left\|T_{N}-T\left(\theta_{M}\right)-T\left(\theta_{M}^{k}\right)\right\|_{\infty} \leq\left\|T_{2 N}^{1}\right\|_{\infty}+\left\|T_{2 N}^{2}-T\left(\theta_{M}^{k}\right)\right\|_{\infty} \\
\leq C_{4} \lambda\left\|T_{N}-T\left(\theta_{M}\right)\right\|_{\psi}+C_{6} \sqrt{a_{k}(2 N, M)}\left\|T_{2 N}^{2}\right\|_{2} .
\end{gathered}
$$

From (1.3) and (1.4) we obtain

$$
\begin{aligned}
\| T_{N} & -T\left(\theta_{M}\right)-T\left(\theta_{M}^{k}\right) \|_{\infty} \\
& \leq \sqrt{\frac{N}{2 M}}\left\|T_{N}\right\|_{2}\left(\sqrt{2} C_{4} C_{3} \lambda+C_{3} C_{5} C_{6} \sqrt{2 a_{k}(2 N, M)} e^{-\lambda^{2}}\right) .
\end{aligned}
$$

Set $\lambda=\sqrt{1 / 2 \log a_{k}(N, 2 M)}$. Then the right-hand side can be rewritten in the form

$$
\left(\frac{N}{2 M} \log a_{k}(N, 2 M)\right)^{1 / 2}\left\|T_{N}\right\|_{2}\left(C_{4} C_{3}+C_{3} C_{5} C_{6} \frac{\sqrt{2 a_{k}(2 N, M)}}{\sqrt{a_{k}(N, 2 M)} \sqrt{\log a_{k}(N, 2 M)}}\right) .
$$

As $\frac{N}{2 M} \log a_{k}(N, 2 M)=a_{k+1}(N, 2 M)$, we need only prove that the expression in parentheses does not exceed an absolute constant $C_{6}$. To this end we show that for every $k$

$$
\frac{2 a_{k}(2 N, M)}{a_{k}(N, 2 M)} \leq 4 \log 4\left(1+\frac{1}{\log N / 2 M}+\ldots+\frac{1}{(\log N / 2 M)^{k-1}}\right) .
$$

For $k=1$

$$
\frac{a_{1}(2 N, M)}{a_{1}(N, 2 M)}=\frac{4 \log 2 N}{\log N} \leq 4 \log 4
$$

Assume that (1.8) holds for $k-1$. Then, according the definition for $k$

$$
\begin{aligned}
& \frac{a_{k}(2 N, M)}{a_{k}(N, 2 M)}=\frac{4 \log a_{k-1}(2 N, M)}{\log a_{k-1}(N, 2 M)}=4\left(1+\frac{1}{\log a_{k-1}(N, 2 M)} \log \frac{a_{k-1}(2 N, M)}{a_{k-1}(N, 2 M)}\right) \\
& \leq 4\left(1+\frac{1}{\log N / 2 M} \log \left(4 \log 4\left(1+\frac{1}{\log N / 2 M}+\ldots+\frac{1}{(\log N / 2 M)^{k-2}}\right)\right.\right. \\
& \leq 4 \log 4\left(1+\frac{1}{\log N / 2 M}+\ldots+\frac{1}{(\log N / 2 M)^{k-1}}\right) .
\end{aligned}
$$

Therefore if $N \geq 2 M e^{2}$, then there exists an absolute constant $C_{7}$ such that $\frac{2 a_{k}(2 N, M)}{a_{k}(N, 2 M)} \leq C_{7}$. We can take $C_{6}>C_{2} C_{3}$ and bound $M<\alpha N$ in such a way that the expression in the parentheses in (1.7) does not exceed $C_{6}$.

An odd $M$ is treated similarly.

The theorem is proved for all $M$ and $N$ such that $M \leq \alpha N$.

For $M$ satisfying $\alpha N<M \leq 2 N$ the conclusion of the theorem follows from the above.

The proof of the theorem in the case $2<p<\infty$ is based on the following decomposition lemma, analogous to Lemma 3. 
Lemma 5. Let a trigonometric polynomial $T_{N}$ and $\lambda>0$ be given. Then $T_{N}$ can be represented as the sum of two polynomials $T_{2 N}^{1}$ and $T_{2 N}^{2}, \quad T_{N}=T_{2 N}^{1}+T_{2 N}^{2}$, such that

$$
\left\|T_{2 N}^{1}\right\|_{\infty} \leq C_{8} \lambda^{1 / p}\left\|T_{N}\right\|_{p}, \quad\left\|T_{2 N}^{2}\right\|_{2} \leq C_{9} \lambda^{1 / p-1 / 2}\left\|T_{N}\right\|_{p} .
$$

Now we can prove the theorem for $2<p<\infty$. By the above, there exists a polynomial $T\left(\theta_{M} ; x\right)$ such that

$$
\left\|T_{2 N}^{2}-T\left(\theta_{M} ; x\right)\right\|_{\infty} \leq C_{6} \sqrt{\frac{N}{M} \log \left(\frac{N}{M}+1\right)}\left\|T_{2 N}^{2}\right\|_{2} .
$$

Therefore by (1.9) we have

$$
\left\|T_{N}-T\left(\theta_{M}\right)\right\|_{\infty} \leq C_{10}\left[\lambda^{1 / p}+\lambda^{1 / p-1 / 2}(N / M \log (N / M+1))^{1 / 2}\right]\left\|T_{N}\right\|_{p} .
$$

Putting $\lambda=N / M \log (N / M+1)$, we obtain the desired result.

Remark 1. As one can see from the proof, the interval where the spectrum of the approximating polynomial is located is doubled on every step. But the actual support of the spectrum is contained in the segment $[-2 N, 2 N]$. This follows from Lemma 1.

Remark 2. If $T_{N}$ is the Dirichlet kernel

$$
T_{N}(x)=\sum_{k=-N}^{N} e^{i k x},
$$

then estimate (1.1) can be derived immediately from [G1] or [Sp]. A more accurate estimate of the approximation of kernel (1.10) is proved in [Mr2].

Remark 3. This method of approximation can be used in every space where statements similar to Lemmas 1-4 are valid.

Proof of Lemma 2. Suppose that $\left\|T_{N}\right\|_{2}=1$, fix $N / M \sim 2^{k}$, and write

$$
\begin{gathered}
\sum_{j=-N}^{N} c_{j} e^{i j x}=\sum_{j=-N}^{N} c_{j} \epsilon_{j}^{1} e^{i j x}+\sum_{j=-N}^{N} c_{j}\left(1-\epsilon_{j}^{1}\right) e^{i j x} \\
=\sum_{j=-N}^{N} c_{j} \epsilon_{j}^{1} e^{i j x}+\sum_{j=-N}^{N} c_{j}\left(1-\epsilon_{j}^{1}\right) \epsilon_{j}^{2} e^{i j x}+\sum_{j=-N}^{N} c_{j}\left(1-\epsilon_{j}^{1}\right)\left(1-\epsilon_{j}^{2}\right) e^{i j x} \\
=\sum_{j=-N}^{N} c_{j} \epsilon_{j}^{1} e^{i j x}+\sum_{j=-N}^{N} c_{j}\left(1-\epsilon_{j}^{1}\right) \epsilon_{j}^{2} e^{i j x}+\ldots \\
+\sum_{j=-N}^{N} c_{j}\left(1-\epsilon_{j}^{1}\right) \ldots\left(1-\epsilon_{j}^{k-1}\right) \epsilon_{j}^{k} e^{i j x} \\
+\sum_{j=-N}^{N} c_{j}\left(1-\epsilon_{j}^{1}\right) \ldots\left(1-\epsilon_{j}^{k}\right) e^{i j x},
\end{gathered}
$$

where $\left\{\epsilon_{j}^{m}\right\}_{-N \leq j \leq N, 1 \leq m \leq k}$ are \pm 1 , the signs to be specified. Denoting (1.11) by $\phi(\epsilon, x)$, we deduce from the definition of norm in the Orlicz space $[\mathrm{Kr}]$ and Khinchin's inequality that 


$$
\begin{gathered}
\int\|\phi(\epsilon, x)\|_{\psi(d x)} d \epsilon=\int \inf _{t>0} \frac{1}{t}\left(1+\int_{\mathbb{T}} \psi(t \phi(\epsilon, x)) d x\right) d \epsilon^{1} \ldots d \epsilon^{m} \\
\leq \int d \epsilon^{1} \ldots d \epsilon^{m-1} \inf _{t>0} \frac{1}{t}\left(\int_{\mathbb{T}} \int \psi(t \phi(\epsilon, x)) d \epsilon^{m} d x\right) \\
\leq C_{11} \sum_{m \leq k} \int\left(\sum_{j=-N}^{N}\left|c_{j}\right|^{2}\left|\left(1-\epsilon_{j}^{1}\right)\right|^{2} \ldots\left|\left(1-\epsilon_{j}^{m-1}\right)\right|^{2}\right)^{1 / 2} d \epsilon^{1} \ldots d \epsilon^{m-1} .
\end{gathered}
$$

The Hölder inequality and integration with respect to $\epsilon^{1}, \ldots, \epsilon^{m-1}$ give

$$
\int\|\phi(\epsilon, x)\|_{\psi(d x)} d \epsilon \leq C_{12} 2^{\frac{k}{2}}
$$

and if we let

$$
A_{\epsilon}=\left\{j \in[-N, N] \mid \epsilon_{j}^{1}=\ldots=\epsilon_{j}^{k}=-1\right\}
$$

then

$$
\begin{gathered}
\left|A_{\epsilon}\right|=\frac{1}{2^{k}} \sum_{j=-N}^{N}\left(1-\epsilon_{j}^{1}\right) \ldots\left(1-\epsilon_{j}^{k}\right), \\
\int\left|A_{\epsilon}\right| d \epsilon=\frac{N}{2^{k}} \sim M .
\end{gathered}
$$

Now (1.12) and (1.13) allow us to choose the signs $\epsilon_{j}^{m}$ so that

$$
T\left(\theta_{M} ; x\right)=\sum_{j}\left(1-\epsilon_{j}^{1}\right) \ldots\left(1-\epsilon_{j}^{k}\right) e^{i j x}
$$

satisfies the condition

$$
\left\|T_{N}(x)-T\left(\theta_{M} ; x\right)\right\|_{\psi} \leq C_{13}\left(\frac{N}{M}\right)^{1 / 2} .
$$

Using the homogeneity of the norm, we conclude the proof of the lemma.

\section{APPROXIMATION IN THE NORM OF UNIFORM CONVERGENCE}

Let $U$ be the Banach space of continuous functions defined on $\mathbb{T}$ with the norm $\|f\|_{U}=\sup _{k}\left\|S_{k}(f)\right\|_{\infty}$, where $S_{k}(f), \quad k=1,2, \ldots$, are the partial sums of the Fourier series of $f$.

Theorem 2. Let $2<p<\infty, 1 \leq M \leq 2 N$, and let $T_{N}(x)$ be a trigonometric polynomial of degree $N$. Then there exists a trigonometric polynomial with a number of harmonics $\leq M$, such that

$$
\left\|T_{N}-T\left(\theta_{M}\right)\right\|_{U} \leq C_{1}\left(\frac{N}{M}\right)^{1 / p}\left(\log \left(\frac{N}{M}+1\right)\right)^{3 / p}\left\|T_{N}\right\|_{p}
$$

and the spectrum $\theta_{M}$ is contained in the segment $[-N, N]$.

In order to use the method of Theorem 1, we need the following results.

Lemma 6. Let $\lambda>1$. Then every polynomial $T_{N}$ can be represented as a sum of two polynomials $T_{N}^{1}, T_{N}^{2}$, of degree at most $N$, such that

$$
\left\|T_{N}^{1}\right\|_{U} \leq C_{2} \lambda\left\|T_{N}\right\|_{\psi}, \quad\left\|T_{N}^{2}\right\|_{2} \leq C_{3} \lambda^{1 / 3} e^{-\lambda^{2 / 3}}\left\|T_{N}\right\|_{\psi} .
$$


Proof. By Lemma $3, T_{N}$ can be represented in the form $T_{N}=T_{2 N}^{1}+T_{2 N}^{2}$, where

$$
\left\|T_{2 N}^{1}\right\|_{\infty} \leq C_{4} t\left\|T_{N}\right\|_{\psi}, \quad\left\|T_{2 N}^{2}\right\|_{2} \leq C_{5} e^{-t^{2}}\left\|T_{N}\right\|_{\psi} .
$$

By [Ki], the function $T_{2 N}^{1}$ may be decomposed into $T_{2 N}^{1}=g+h$, where

$$
\|g\|_{U} \leq C_{6} \mu\left\|T_{2 N}^{1}\right\|_{\infty}, \quad\|h\|_{2} \leq C_{7} e^{-\mu}\left\|T_{2 N}^{1}\right\|_{\infty} .
$$

Therefore $T_{N}=g+T_{2 N}^{2}+h$, where

$$
\|g\| \leq C_{8} t \mu\left\|T_{N}\right\|_{\psi}, \quad\left\|T_{2 N}^{2}+h\right\|_{2} \leq C_{9}\left(e^{-t^{2}}+t e^{-\mu}\right)\left\|T_{N}\right\|_{\psi} .
$$

We put now $t=\lambda^{1 / 3}$ and $\mu=\lambda^{2 / 3}$. By the uniform boundedness of the operators of partial sums in the space $U$, the lemma follows.

Lemma 7. Let $1 \leq M \leq 2 N$, and let $T_{N}$ be a trigonometric polynomial of degree $\leq N$. Then there exists a trigonometric polynomial $T\left(\theta_{M}\right)$, with a number of harmonics $\leq M$, such that

$$
\left\|T_{N}-T\left(\theta_{M}\right)\right\|_{U} \leq C_{10}\left(\frac{N}{M} \log N\right)^{1 / 2}\left\|T_{N}\right\|_{2} .
$$

The proof is analogous to that of Lemma 4.

Now the proof of Theorem 2 follows as Theorem 1 . We consider first the case $p=2$. The iterative sequence $(R=N / M)$

$$
a_{1}=R \log N, \quad a_{2}=R \log ^{3} a_{1}, \ldots a_{k+1}=R \log ^{3} a_{k}, \ldots
$$

converges to $x_{0}$ the largest root of the equation $x=R \log ^{3} x$ which can be estimated by $R \log ^{3} R<x_{0}<2 R \log ^{3} R$. After a finite number of steps, the desired result is achieved.

Let $2<p<\infty$. The following result can be proved by the same method as in [Ki2]

Lemma 8. Let $\lambda>0$ and $2<p<\infty$. Then every polynomial $T_{N}$ can be represented as a sum of two polynomials $T_{N}^{1}$ and $T_{N}^{2}$, of degree at most $N$, such that

$$
\left\|T_{N}^{1}\right\|_{U} \leq C_{11} \lambda^{1 / q}\left\|T_{N}\right\|_{q}, \quad\left\|T_{N}^{2}\right\|_{2} \leq C_{12} \lambda^{1 / q-1 / 2}\left\|T_{N}\right\|_{q} .
$$

Using this lemma and the already-proved result for $p=2$, we obtain the assertion of the theorem.

Remark 4. Theorem 2 gives a better estimate than Lemma 7 only for $M$ close to $N$.

Remark 5. The essential part of the proof is the decomposition obtained in Lemma 6 , the proof of which is based on Carleson's famous theorem about convergence almost everywhere of the Fourier series for functions in $L_{2}$. It would be interesting to give another proof independent of this deep result.

\section{Approximation of ANALYTIC POLYNOMials in the SPACE $H^{\infty}$}

Let us consider an analytic polynomial, i.e.

$$
T_{N}(x)=\sum_{k=0}^{N} c_{k} e^{i k x}
$$

and estimate its best approximation by analytic polynomials with $M$ harmonics. Note that we cannot use Theorem 1 because the polynomial in Theorem 1 is not 
analytic. It happens that for an analytic polynomial $T_{N}$, the approximating polynomial in Theorem 2 is analytic. However Theorem 2 yields a worse estimate of the approximation.

Theorem 3. Let $T_{N}$ be an analytic trigonometric polynomial, and let $2 \leq p<$ $\infty$ and $1 \leq M \leq N$. Then there exists an analytic trigonometric polynomial $T\left(\theta_{M} ; x\right)$, with a number of harmonics $\leq M$, such that

$$
\left\|T_{N}-T\left(\theta_{M}\right)\right\|_{\infty} \leq C_{1}\left(\frac{N}{M} \log \left(\frac{N}{M}+1\right)\right)^{1 / p}\left\|T_{N}\right\|_{p}
$$

and $\theta_{M} \subset[0,2 N]$.

We begin with the case $p=2$. The main lemma to be proved is the decomposition lemma.

Lemma 9. Let $\lambda>0$ be given. Every analytic polynomial can be represented as the sum of two analytic polynomials, $T_{N}=T_{2 N}^{1}+T_{2 N}^{2}$, of degree $\leq 2 N$, such that

$$
\left\|T_{2 N}^{1}\right\|_{\infty} \leq C_{2} \lambda\left\|T_{N}\right\|_{\psi}, \quad\left\|T_{2 N}^{2}\right\|_{2} \leq C_{3} e^{-\lambda^{2}}\left\|T_{N}\right\|_{\psi}
$$

Proof. We follow [Ki1] and assume without loss of generality that $\left\|T_{N}\right\|_{\psi}=1$. We truncate $T_{N}$ by multiplying it by an analytic function $\tau(x)=[\alpha+i H(\alpha)]^{-1}$, where $H$ is the Hilbert transform and $\alpha(x)=\max \left(1, \frac{\left|T_{N}\right|}{\lambda}\right)$. It is obvious that $|\tau(x)| \leq 1 / \alpha(x)$; therefore $\left|T_{N}(x) \tau(x)\right| \leq \lambda$. From the definition of the norm in the Orlicz space and the Tchebychev inequality we have

$$
\mu\left\{x:\left|T_{N}(x)\right|>\lambda\right\} \leq e^{-\lambda^{2} / 2} .
$$

Denote $E_{\lambda}=\left\{x:\left|T_{N}\right|>\lambda\right\}$. Then

$$
\int_{\mathbb{T}}\left|(1-\tau(x)) T_{N}(x)\right|^{2} d x \leq C_{4}\left(\int_{\mathbb{T}}\left|T_{N}\right|^{2} \frac{|1-\alpha|^{2}}{\alpha^{2}} d x+\int_{\mathbb{T}}\left|T_{N}\right|^{2} \frac{\left.H(1-\alpha)\right|^{2}}{\alpha^{2}} d x\right) .
$$

Because $\alpha(x)=1$ if $\left|T_{N}\right| \leq \lambda$, the first term in (3.4) can be rewritten in the form $\int_{E_{\lambda}}|| T_{N}|-\lambda|^{2} d x$, which by virtue of (3.3) can in turn be estimated by

$$
\int_{\lambda}^{\infty}|u-\lambda| e^{-u^{2} / 2} d u \leq C_{5} e^{\lambda^{2} / 2}
$$

For the second term we have

$$
\int_{\mathbb{T}}\left|T_{N}\right|^{2} \frac{|H(1-\alpha)|^{2}}{\alpha^{2}} d x \leq \lambda^{2} \int_{\mathbb{T}}|H(1-\alpha)|^{2} d x .
$$

Since the Hilbert transform is bounded in $L_{2}$, it follows that

$$
\int_{\mathbb{T}}|H(1-\alpha)|^{2} d x \leq \int_{\mathbb{T}}(1-\alpha)^{2} d x=\int_{\left|T_{N}\right|>\lambda}\left(1-\left|T_{N}\right| / \lambda\right)^{2} d x,
$$

which we have already estimated. Now $T_{N}=\tau T_{N}+(1-\tau) T_{N} \equiv f_{1}+f_{2}$, where $f_{1}$ and $f_{2}$ are analytic functions. We apply the de la Vallée-Poussin operator and Lemma 1 , and the proof follows.

Now the theorem for $p=2$ can be proved by the method of Theorem 1 . We omit the details.

Since for $2<p<\infty$ every analytic polynomial can be decomposed as $T_{N}=$ $T_{2 N}^{1}+T_{2 N}^{2}$ with $\left\|T_{2 N}^{1}\right\|_{\infty} \leq C_{6} \lambda^{1 / p}\left\|T_{N}\right\|_{p}, \quad\left\|T_{2 N}^{2}\right\|_{2} \leq C_{7} \lambda^{1 / p-1 / 2}\left\|T_{N}\right\|_{p}$ (see [Ki2]), it is sufficient to apply the statement for $p=2$ and choose an optimal $\lambda$. 


\section{Applications}

1. Best approximation by trigonometric polynomials of functions from the Sobolev classes. We consider $W_{p}^{r, \alpha}(1 \leq p<\infty)$, the class of functions which have the integral representation

$$
f(x)=\frac{1}{2 \pi} \int_{\mathbb{T}} \phi(x-u) F_{r, \alpha}(u) d u,
$$

where

$$
F_{r, \alpha}(u)=\sum_{k=1}^{\infty} \frac{1}{k^{r}} \cos (k u-\pi \alpha / 2), \quad 0 \leq \alpha<2,
$$

is a Bernoulli kernel and $\|\phi\|_{p} \leq 1$. We consider

$$
e_{m}\left(W_{p}^{2, \alpha} ; X\right)=\sup _{f \in W_{p}^{r, \alpha}} e_{m}(f ; X),
$$

the error of the best approximation in the Banach space $X$ of the class $W_{p}^{r, \alpha}$ by trigonometric polynomials with a prescribed number of harmonics.

Theorem 4. Let $1 \leq p \leq \infty$ and $r>1 / p$. Then

$$
e_{m}\left(W_{p}^{r, \alpha} ; U\right) \asymp m^{-\min (r, r-1 / p+1 / 2)} .
$$

The lower estimate follows immediately from [Be1], since

$$
e_{m}\left(W_{p}^{r, \alpha} ; U\right) \geq e_{m}\left(W_{p}^{r, \alpha} ; L_{2}\right) .
$$

The upper estimate can be proved in the usual way (see for example [Be2]).

The exact order of $e_{m}\left(W_{p}^{r, \alpha} ; L_{q}\right), 2<q<\infty$, was found earlier in [Be2].

Corollary. Let $1 \leq p \leq \infty$ and $r>1 / p$. Then

$$
e_{m}\left(W_{p}^{r, \alpha} ; L_{\infty}\right) \asymp m^{-\min (r, r-1 / p+1 / 2)} .
$$

2. Best approximation of analytic functions in the unit disk $|z| \leq 1$. In our approach to this problem we consider the subspace $W_{A, p}^{r, \alpha} \subset W_{p}^{r, \alpha}$ of functions of the form (4.1) with Fourier series containing only exponentials with positive indices.

Theorem 5. Let $1 \leq p \leq \infty$ and $r>1 / p$. Then

$$
e_{m}\left(W_{A, p}^{r, \alpha} ; H^{\infty}\right) \asymp m^{-\min (r, r-1 / p+1 / 2)} .
$$

The proof is based on Theorem 3 .

3. Best approximation and the estimate of trigonometric widths for the classes of functions of several variables. Let $f$ be a real function on $\mathbb{T}^{n}=[-\pi, \pi]^{n}, 2 \pi$-periodic in each variable, and let $r$ be an integer. Let $W_{p}^{r}$ be the class of functions $f$ with

$$
\sum_{r_{1}+r_{2}+\ldots+r_{n}=r}\left\|D_{x_{1}}^{r_{1}} D_{x_{2}}^{r_{2}} \ldots D_{x_{n}}^{r_{n}} f\right\|_{p} \leq 1 .
$$

We shall estimate the trigonometric widths

$$
d_{m}^{T}\left(W_{p}^{r} ; L_{q}\right) \stackrel{\text { def }}{=} \inf _{\theta_{m}} \sup _{f \in W_{p}^{r}} \inf _{T\left(\theta_{m}\right)}\left\|f-T\left(\theta_{m}\right)\right\|_{q},
$$


where the infimum is taken over all subsets of cardinality $m$ in $\mathbb{Z}^{n}$. It is obvious that the trigonometric $m$-width is no less than the Kolmogorov $m$-width, namely,

$$
d_{m}\left(W_{p}^{r} ; L_{q}\right) \stackrel{\text { def }}{=} \inf _{L_{m}} \sup _{f \in W_{p}^{r}} \inf _{u \in L_{m}}\|f-u\|_{q}
$$

where the infimum is taken over all subspaces $L_{m}$ of dimension $m$.

Another obvious but useful inequality is

$$
e_{m}\left(W_{p}^{r} ; L_{q}\right) \leq d_{m}^{T}\left(W_{p}^{r} ; L_{q}\right)
$$

Theorem 6. Let $1 \leq p \leq 2, p^{\prime}=p / p-1,2<q \leq p^{\prime} \leq \infty$, and $r>n$. Then

$$
d_{m}^{T}\left(W_{p}^{r} ; L_{q}\right) \asymp m^{-(r / n-1 / p+1 / 2)} .
$$

Due to estimates for the Kolmogorov widths ([Mr3], [Ho]), the lower estimate is a corollary of them. The upper estimate strengthens the corresponding estimate for the Kolmogorov widths. The proof of the upper estimate is based on the extension of the estimate of Theorem 1 to the case of several variables.

Remark 6. In this connection it would be interesting to find an extension of Theorem 4 to the case of several variables. The main open question is a corresponding decomposition lemma.

\section{Acknowledgments.}

I would like to express my deep gratitude to Yuly Makovoz and Dany Leviatan for their helpful comments on this paper. I also thank the referee, who pointed out to me how Lemma 8 and therefore Theorem 2 could be strengthened.

Note. After this paper was submitted the author learned that a result analogous to Theorem 1 was proved by another method by R. DeVore and V. Temlyakov. Their proof is mainly based on Gluskin's finite-dimensional result [G1].

\section{REFERENCES}

[St] S. B. Stechkin, On the best approximation of given classes of functions by arbitrary polynomials, Uspekhi Matematicheskikh Nauk 9 (1) (1954), 133-134 (Russian).

[Is] R. S. Ismagilov, Widths of set in normed linear spaces and approximation of functions by trigonometric polynomials, Uspecki Matematicheskikh Nauk 29 (3) (1974), 161-178 (Russian), English translation in Russian Math. Surveys 28 (3) (1974). MR 53:11284

[Mr1] V. E. Maiorov, On linear widths of Sobolev classes and chains of extremal subspaces, Matematicheskiŭ Sbornik 113 (1980), 437-463; 119 (1982), 301; English translations in Math. USSR Sb. 41 (1982); 47 (1984). MR 82j:41022; MR 84b:41021

[Mr2] V. E. Maiorov, Trigonometric widths of Sobolev classes $W_{p}^{r}$ in the space $L_{q}$, Matematicheskie Zametki 40 (2) (1986), 161-173; English translation in Math. Notes 40 (1986). MR 87k: 46072

[Mr3] V. E. Maiorov, On the best approximation of classes $W_{1}^{r}\left(I^{s}\right)$ in the space $L_{\infty}\left(I^{s}\right)$, Matematicheskie Zametki 19 (1976), 699-706; English translation in Math. Notes 19 (1976). MR 54:10946

[Mk] Y. Makovoz, On trigonometric n-widths and their generalizations, J. Approx. Theory 41 (1984), 361-366. MR 86g:41038

[Be1] E. S. Belinskii, Approximation of periodic functions by a "floating" system of exponentials, Studies in the Theory of Functions of Several Real Variables (Y. A. Brudnyi, ed.), Yaroslav. Gos. Univ., Yaroslavl, 1984, pp. 10-24 (Russian). MR 88j:42002

[Be2] E. S. Belinskii, Approximation by a "floating" system of exponentials on classes of smooth periodic functions, Matematischeskiü Sbornik 132 (1987), 20-27; English translation in Math. USSR Sb. 60 (1988). MR 88d:42001 
[Z] A. Zygmund, Trigonometric series, 2nd ed., Cambridge Univ. Press, Cambridge, 1959. MR 21:6498

[Bo] Y. Bourgain, Bounded orthogomal systems and the $\Lambda(p)$-set problem, Acta Math. 162 (3-4) (1989), 227-245. MR 90h:43008

[G1] E. D. Gluskin, Extremal properties of orthogonal parallelepipeds and their application to the geometry of Banach spaces, Matematischeskiǔ Sbornik 136 (1988), 85-96; English translation in Math. USSR Sb. 64 (1989). MR 89j:46106

[Sp] J. Spencer, Six standard deviations suffice, Trans. Amer. Math. Soc. 289 (2) (1985), 679706. MR 86k:05005

[Ki1] S. V. Kislyakov, Quantitative aspect of the "corrigible" theorems, Investigations on Linear Operators and Function Theory, Zapiski LOMI 92 (1979), 182-191. (Russian) MR 82c: 28012

[Ki2] S. V. Kislyakov, Fourier coefficints of boundary values of functions that are analytic in the disk and bidisk, Spectral Theory and Functional Operators II, Trudy Math. Inst. Steklov 155 (1981) 77-94; English translation in Proc. Steklov Inst. Math. 1983, no. 1 (155). MR 83a: 42005

[Ho] K. Höllig, Approximationszahlen von Sobolev-Einbettungen, Mathematische Annalen 242 (1979), 273-281. MR 80j:46051

[Kr] M. A. Krasnoselskii and Y. B. Rutitskii, Convex functions and Orlicz spaces, Fizmatgiz, Moscow, 1958; English translation, Noordhoff, Groningen, 1961. MR 21:5144; MR 23:A4016

Department of Mathematics, Technion, 32000, Haifa, Israel

Current address: Department of Mathematics, University of Zimbabwe, P. O. Box MP167, Mount Pleasant, Harare, Zimbabwe

E-mail address: belinsky@maths.uz.zw 